Pacific Journal of Mathematics

KLEIN-GORDON SOLVABILITY AND THE GEOMETRY O

John KeLly Beam AND PhilLip E. PARKer 


\title{
KLEIN-GORDON SOLVABILITY AND THE GEOMETRY OF GEODESICS
}

\author{
John K. BeEm ANd Phillip E. PARKer
}

The Klein-Gordon equation is globally solvable on Lorentzian manifolds which have no imprisoned causal geodesics and which satisfy a certain convexity condition: for each compact subset $K$ there exists a compact subset $K^{\prime}$ such that any causal geodesic segment with both endpoints in $K$ lies in $K^{\prime}$. This collection of Lorentzian manifolds includes many which are not globally hyperbolic. In any such manifold, the causal convex hull of a compact set is compact. When a curvature condition is satisfied, causally related points can be joined by at least one causal geodesic. A large class of these manifolds which fail to be globally hyperbolic may be constructed using warped products. The construction is independent of the warping function.

1. Introduction. In this paper we shall study Lorentzian geometries in which the inhomogeneous wave equation is globally solvable. Spacetimes which are globally hyperbolic comprise a class of examples of such geometries, but the class we shall study is much larger. Perhaps the simplest example which is not globally hyperbolic is a strip parallel to the time axis in the Minkowski plane.

Let $(X, \beta)$ be a Lorentzian manifold. Here we take $\beta$ to be a $(2,0)$-tensor, rather than the more usual $(0,2)$-tensor, for reasons which we now explain. The d'Alembertian operator is $\square:=\operatorname{div} \operatorname{grad}: C^{\infty}(X) \rightarrow$ $C^{\infty}(X)$. By polarization we may regard $\beta \in C^{\infty}\left(T^{*} X\right)$, so that the principal symbol of $\square$ may be identified as $\beta$. Recall that $T^{*} X$ is a symplectic manifold in a natural way. For the discussion of global solvability, certain integral curves of the Hamiltorian vector field $H^{\beta}$ play the decisive role. These are the bicharacteristic strips, those along which $\beta=0$. Their projections in $X$ are the bicharacteristics and are, up to parametrization, the null geodesics of $\beta$. When $\beta$ is not smooth, this is vital [11].

To have any hope of solving the inhomogeneous wave equation

$$
\square u=f
$$

we must allow $u$ to be distributional (in the sense of Schwartz), and then we might as well let $f$ be distributional too. In general, there are distributions $\mathscr{Q}^{\prime}(X)$ and twisted distributions $\mathscr{Q}_{1}^{\prime}(X)$. We shall use the natural 
density $|\operatorname{det} \beta|^{1 / 2}$ to identify $\mathscr{Q}^{\prime}(X)$ and $\mathscr{Q}_{1}^{\prime}(X)$. Thus we extend $\square$ as usual and regard

$$
\square: \mathscr{D}^{\prime}(X) \rightarrow \mathscr{Q}^{\prime}(X) .
$$

Ideally, one would like to have an inverse for $\square$. Unfortunately $\square$ is not invertible, however, so one settles for a parametrix, an inverse modulo $C^{\infty}(X)$. This is an operator $E: \mathscr{Q}^{\prime}(X) \rightarrow \mathscr{Q}^{\prime}(X)$ such that $E \square=\square E=$ [identity on $\mathscr{Q}^{\prime}(X) / C^{\infty}(X)$ ]. Taking $u=E f$, we find $\square u=\square E f \in f+$ $C^{\infty}(X)$. A parametrix is thus sufficient for the study of propagation of singularities, and there are in fact standard techniques for solving specific problems with them. The classical literature on partial differential equations is full of these, and we mention [5] as a modern exposition for the wave equation, and the references there. See also [3] and the references there.

We say that $\square$ is of principal type iff no complete bicharacteristic stays in a compact set. We say that $X$ is $\square$-pseudoconvex iff for every compact $K \subseteq X$ there exists a compact $K^{\prime} \subseteq X$ such that any bicharacteristic interval with both endpoints in $K$ lies wholly in $K^{\prime}$. As a special case of the theorem of Duistermaat and Hörmander, we have that for $\square$ of principal type, there exists a global parametrix iff $X$ is $\square$-pseudoconvex. Therefore, we shall study Lorentzian geometries $(X, \beta)$ in which $\square$ is of principal type and $X$ is $\square$-pseudoconvex (see $\S 2$ for precise conditions).

When $X$ is a spacetime, being of principal type is slightly stronger than being causal; e.g., distinguishing implies principal type. Similarly, global hyperbolicity implies $\square$-pseudoconvexity. We shall show that there are Lorentzian manifolds (even spacetimes!) which are $\square$-pseudoconvex but not globally hyperbolic. Thus we shall be studying a large new class of Lorentzian manifolds (and spacetimes) in which one has available all the machinery for solving wave and Klein-Gordon equations formerly found only in globally hyperbolic manifolds.

We now turn to a brief description of the contents. Section 2 is preliminary, and contains our notations, sign conventions, and definitions.

In $\$ 3$ our main result is that the causal convex hull of a compact set is itself compact (Theorem 3.4) in our Lorentzian geometries.

Section 4 consists of generalizations to Lorentzian manifolds of several results known for globally hyperbolic spacetimes. The main result here is that under an additional assumption on curvature, all pairs of causally related points can be joined by a causal geodesic.

Finally, $\$ 5$ contains various results about warped products. These manifolds have been extremely useful in General Relativity (see §2). In 
particular, we show that any warped product of a globally hyperbolic spacetime with a geodesically pseudoconvex (see \$2) Riemannian manifold is one of our Lorentzian geometries.

We would like to acknowledge a helpful conversation with Bent Petersen concerning the condition of principal type.

2. Conventions. All manifolds considered herein are smooth, meaning $C^{\infty}$, connected and paracompact. Let $X$ be an $n$-dimensional manifold and $\beta$ a nondegenerate symmetric $(2,0)$-tensor on $X$. $\beta$ is called a pseudoriemannian structure or metric tensor. Although one usually uses a $(0,2)$-tensor, for reasons given in the introduction we use a $(2,0)$-tensor. If $\beta$ is positive-definite it is called Riemannian and if the signature of $\beta$ is $n-2$ or $2-n$ it is called Lorentzian. We shall use $\beta$ of signature $2-n$, $(+-\cdots-)$, since historically the wave equation is given by

$$
\left(\frac{\partial^{2}}{\partial t^{2}}-\sum_{1}^{n} \frac{\partial^{2}}{\partial x_{i}^{2}}\right) u=0 .
$$

Since $\beta$ is nondegenerate it induces isomorphisms

$$
\beta^{\sharp}: T^{*} X \rightarrow T X \text { and } \beta^{b}: T X \rightarrow T^{*} X
$$

referred to as raising and lowering indices, respectively. A covector $\xi$ is called timelike, causal, null (or lightlike), or spacelike iff $\beta(\xi, \xi)>0, \geq 0$, $=0$, or $<0$, respectively. The classification is extended to vectors via $\beta$; e.g., a vector $v$ is causal iff $\bar{\beta}(v, v):=\beta\left(\beta^{b}(v), \beta^{b}(v)\right) \geq 0$. It is further extended to 1-forms, vector fields, and curves in the obvious pointwise way. The classification induces a splitting (unnatural) $T^{*} X=t^{*} X \oplus s^{*} X$ into complementary subbundles of timelike and spacelike covectors, respectively. $(X, \beta)$ is called time- or space-orientable according as $t^{*} X$ or $s^{*} X$ is orientable, respectively. If $(X, \beta)$ is Lorentzian, time-orientability is equivalent to the existence of a nonvanishing timelike 1-form, or by raising indices, a nonvanishing timelike vector field [1, p. 16f]. A pseudoriemannian manifold which is not time-orientable has a two-fold cover which is: see [1, p. 19] for the Lorentzian case; the general case may be handled by the usual methods of algebraic topology. If $X$ is time-orientable one may make it time-oriented by choosing a time-orientation, and similarly for space-orientable pseudoriemannian manifolds. We shall call a time-oriented Lorentzian manifold a spacetime, the chosen direction the future, and the other the past.

For $x \in X, I(x)$ is the chronological domain of $x$ which consists of all points that can be joined to $x$ by a timelike curve. Similarly, $J(x)$ is the 
causal domain of $x$ defined by replacing "timelike" with "causal" (including $x$ as as degenerate causal curve). $X$ is said to be chronological iff there are no closed timelike curves and causal iff there are no nondegenerate closed causal curves. It is strongly causal iff each point has arbitrarily small neighborhoods such that no causal curve which leaves one ever returns to it. $X$ is weakly distinguishing iff $I(x)=I(y)$ implies $x=y$. A spacetime allows a decomposition $I(x)=I^{+}(x) \cup I^{-}(x)$ with $I^{+}(x) \cap$ $I^{-}(x)=\varnothing$ when it is chronological, and also $J(x)=J^{+}(x) \cup J^{-}(x)$ with $J^{+}(x) \cap J^{-}(x)=\{x\}$ when it is causal. Here + and - refer to future and past, respectively. In a spacetime, weakly distinguishing may be refined into future and past distinguishing, and a spacetime which is both is called strongly distinguishing. When no confusion arises, we shall use distinguishing to mean weakly distinguishing for Lorentzian manifolds and strongly distinguishing for spacetimes.

Let $(X, \beta)$ be distinguishing. It is called causally simple iff each $J(x)$ is closed. If $I$ is outer continuous as a set-valued function [1, p. 24] $X$ is said to be causally continuous [7]. ( $I$ is always inner continuous.)

Finally, $(X, \beta)$ is stably causal iff there is a neighborhood of $\beta$ in the $C^{0}$-fine (or -Whitney) topology on the space of all Lorentzian structures on $X$, such that all structures in the neighborhood are causal.

Completeness will always mean geodesic completeness. In the positive definite or Riemannian case this is well-known to be equivalent to metric completeness.

We shall also need the warped product construction. Let $(X, \beta)$ and $(Y, b)$ be pseudoriemannian manifolds and $\psi: X \rightarrow(-\infty, 0)$ a smooth function. We define

$$
X \times_{\psi} Y:=(X \times Y, \beta \oplus \psi b) .
$$

$\psi$ is called the warping function. If the first factor is Lorentzian and the second Riemannian, the warped product is Lorentzian. See [10] for general warped products and [1] for Lorentzian warped products. Examples of Lorentzian products which have been useful in General Rélativity include Einstein's static universe and Minkowski, Schwarzschild, de Sitter and Robertson-Walker spacetimes.

Now let $P$ be a scalar pseudodifferential operator of order $r$ on $X$ with principal symbol $p$. We denote the smooth functions on $X$ by $\mathcal{E}(X)$ and those with compact support by $\mathscr{D}(X)$, the distributions by $\mathscr{D}^{\prime}(X)$ and those with compact support by $\mathcal{E}^{\prime}(X)$. Recall that $p \in \mathcal{E}\left(T^{*} X \backslash 0\right)$ and is homogeneous of degree $r$ in the fiber variables. When $(X, \beta)$ is Lorentzian and $P$ is the d'Alembertian $\square:=\operatorname{div} \operatorname{grad}, p$ is the tensor $\beta$ considered as 
an element of $\mathcal{E}\left(T^{*} X \backslash 0\right)$ via $\beta(\xi):=\beta(\xi, \xi)$. Also recall that $T^{*} X \backslash 0$ is a natural symplectic manifold with respect to the canonical 2-form $\omega$ [9]. The Hamiltonian vector field $H^{p}$ is defined by

$$
d p=\omega\left(H^{p},\right)
$$

and $p$ is constant along the integral curves of $H^{p}$. Those along which $p=0$ are called bicharacteristic strips of $P$, and their projections in $X$, bicharacteristics of $P$. The projections of integral curves of $H^{\beta}$ are pregeodesics: after a change of parameter they are geodesics of $\beta$. Thus the bicharacteristics of the d'Alembertian are essentially the null geodesics. Bicharacteristics of an operator describe the propagation of singularities created by the operator [3, Theorem 6.1 .1 ; 13, Chapters IX and X]. The geometry of the bicharaceristics determines global solvability for the equation

$$
P u=f
$$

where $f \in \mathscr{Q}^{\prime}(X)$.

To explain this, recall that $P$ is of real principal type iff $p$ is real-valued and no complete bicharacteristic stays in a compact set. In the terminology of [6], there are no imprisoned bicharacteristics. $X$ is $P$-pseudoconvex iff for every compact $K \subseteq X$ there exists a compact $K^{\prime} \subseteq X$ such that every bicharacteristic segment with both endpoints in $K$ lies in $K^{\prime}$.

TheOREM [3, Theorems 6.3.3 and 6.5.3]. Let $P$ be of real principal type. There exists a global two-sided parametrix for $P$ iff $X$ is $P$-pseudoconvex.

We shall study metric tensors satisfying these two conditions and slight variations thereof. We shall say that $(X, \beta)$ is causally pseudoconvex iff $X$ is $\left(\square-m^{2}\right)$-pseudoconvex for all $m \geq 0$, and principally causal iff $\square-m^{2}$ is of principal type for all $m \geq 0$. If $(X, \beta)$ is Riemannian we shall say that it is geodesically pseudoconvex iff for every compact $K \subseteq X$ there exists a compact $K^{\prime} \subseteq X$ such that every geodesic segment with both endpoints in $K$ lies in $K^{\prime}$.

Causally pseudoconvex Lorentzian manifolds have been used in a proof of the existence of Hawking radiation from spacetime singularities [12]. Since the bicharacteristics of the Laplacian are points, every Riemannian manifold is $\Delta$-pseudoconvex. Geodesic pseudoconvexity is a natural variant, and is studied in $\$ 5$. Principal causality is equivalent to the condition that no inextendible causal geodesic lies in a compact set. Indeed, any integral curve of a (fiber homogeneous) vector field which stays in (or over) a compact set is complete. However, the coordinates 
used for geodesics may destroy compactness; i.e., they are not necessarily smoothly related to induced bicharacteristics coordinates.

3. The causal convex hull. It is well known [6, pp. 194ff] that noncompact spacetimes may contain inextendible causal geodesics which are future (or past) imprisoned. In this section we show that a spacetime contains an inextendible causal geodesic which is future (or past) imprisoned iff it contains one which is both future and past imprisoned. Recall (§2) that Lorentzian manifolds which do not contain an imprisoned inextendible causal geodesic are principally causal. Distinguishing and strongly causal spacetimes are always principally causal, but causal spacetimes need not be $[\mathbf{1} ; \mathbf{6}]$.

Let $(X, \beta)$ be a Lorentzian manifold and $K \subseteq X$. The causal convex hull of $K, \llbracket K \rrbracket$ consists of all points which lie in $K$ or on a causal geodesic segment having both endpoints in $K$. In this section we show that if $(X, \beta)$ is principally causal and causally pseudoconvex, then $K$ compact implies $\llbracket K \rrbracket$ compact.

We begin with a technical lemma on manifolds with an affine connection.

LeMMA 3.1. Let $(X, \nabla)$ be a manifold with affine connection. If it has an inextendible geodesic $\gamma:(a, b) \rightarrow X$ with $a<t_{0}<b$ and lying in $K a$ compact subset of $X$ for $t_{0} \leq t<b$, then it has an inextendible geodesic $\tilde{\gamma}$ : $(c, d) \rightarrow X$ which is imprisoned in K. Furthermore, the image of $\tilde{\gamma}$ lies in the closure of the image of $\gamma$.

Proof. Let $h$ be auxiliary complete Riemannian metric on $X$. Since $\lim _{t \rightarrow b} \gamma(t)$ does not exist there are sequences $\left\{t_{n}\right\}$ and $\left\{s_{n}\right\}$ with $t_{n}, s_{n} \rightarrow b^{-}$ and $t_{0}<s_{0}<t_{1}<s_{1}<\ldots$ such that $\gamma\left(t_{n}\right) \rightarrow x_{1}$ and $\gamma\left(s_{n}\right) \rightarrow x_{2}$ where $x_{1} \neq x_{2}$ are points of $K$. Letting $L$ denote $h$-arclength, then $x_{1} \neq x_{2}$ implies that $L\left(\gamma \mid\left[t_{0}, t_{n}\right]\right) \rightarrow \infty$ as $n \rightarrow \infty$. Let $v_{n}$ be the $h$-unit vector in the direction of $\dot{\gamma}\left(t_{n}\right) \in T_{\gamma\left(t_{n}\right)} X$. Since the $h$-unit sphere bundle over $K$ is compact, by passing to a subsequence if necessary we may assume that $v_{n} \rightarrow v$ in the $h$-unit sphere bundle over $K$. Set $\tilde{\gamma}:(c, d) \rightarrow X$ equal to the unique inextendible geodesic of $\nabla$ with $\dot{\gamma}(0)=v$.

For each $n$ let $\gamma_{n}$ be the reparametrization of $\gamma$ such that $\dot{\gamma}_{n}(0)=v_{n}$. Then $\dot{\gamma}_{n}(0) \rightarrow \dot{\gamma}(0)$ as $n \rightarrow \infty$ and for each $t \in(c, d), \gamma_{n}(t) \rightarrow \tilde{\gamma}(t)$. Therefore the image of $\bar{\gamma}$ lies in the closure of the image of $\gamma$. 
Now assume that there exists $\theta \in(c, d)$ with $\tilde{\gamma}(\theta) \notin K$. If $\theta<0$ then the smoothness of $\tilde{\gamma}$ implies that $\tilde{\gamma}[\theta, 0]$ has finite $h$-length. Since $L$ is continuous we find $L\left(\gamma_{n} \mid[\theta, 0]\right) \rightarrow L(\tilde{\gamma} \mid[\theta, 0])<\infty$, whence $L\left(\gamma \mid\left[t_{0}, t_{n}\right]\right)$ $\rightarrow \infty$ yields $\gamma_{n}[\theta, 0] \subseteq \gamma\left[t_{0}, t_{n}\right] \subseteq K$ for large $n$. But then $\tilde{\gamma} \mid[\theta, 0] \subseteq K$, a contradiction. If $\theta>0$ the argument is similar.

COROLlaRy 3.2. These three conditions are equivalent for a spacetime $(X, \beta)$ :

(1) it has an inextendible causal geodesic which is future imprisoned;

(2) it has an inextendible causal geodesic which is past imprisoned;

(3) it has an inextendible causal geodesic which is imprisoned (both ways).

Minkowski spacetime with a single point deleted shows that if $\left\{\gamma_{n}\right\}$ is a sequence of causal geodesic segments from $x_{n}$ to $y_{n}$ and if $x_{n} \rightarrow x$, $y_{n} \rightarrow y$, then there is not necessarily a causal geodesic segment from $x$ to $y$. This example is principally causal but not causally pseudoconvex.

LeMma 3.3. Let $(X, \beta)$ be a principally causal and causally pseudoconvex Lorentzian manifold. If $x \neq y, x_{n} \rightarrow x, y_{n} \rightarrow y$, and for each $n$ there is a causal geodesic segment $\gamma_{n}$ from $x_{n}$ to $y_{n}$, then there is a causal geodesic segment $\gamma$ from $x$ to $y$.

Proof. Let $h$ be an auxiliary Riemannian metric on $X$. We may assume that $\gamma_{n}(0)=x_{n}$ and that $\dot{\gamma}_{n}(0)$ is an $h$-unit vector for each $n$. Let $K$ be a compact set containing $\left\{x_{n}, y_{n}, x, y\right\}$. By causal pseudoconvexity there exists a compact set $K^{\prime} \supseteq \llbracket K \rrbracket$. By compactness and by passing to a subsequence if necessary, we may assume that $\dot{\gamma}_{n}(0) \rightarrow v \in T_{x} X$, an $h$-unit vector. Set $\gamma:[0, b) \rightarrow X$ equal to the unique $\beta$-geodesic which is inextendible to $b$ and satisfies $\dot{\gamma}(0)=v$. Since each $\gamma_{n}$ is causal so is $\gamma$.

Extend each original geodesic segment to $\gamma_{n}:\left[0, a_{n}\right) \rightarrow X$ which is inextendible to $a_{n}$. Principal causality and Lemma 3.1 imply that $\gamma\left(t_{0}\right) \notin$ $K^{\prime}$ for some $t_{0}<b$. Since $\gamma_{n}\left(t_{0}\right) \rightarrow \gamma\left(t_{0}\right)$ it follows that $\gamma_{n}\left(t_{0}\right) \notin K^{\prime}$ for all sufficiently large $n$. Let $t_{n}$ be the smallest number in $\left[0, a_{n}\right)$ such that $\gamma_{n}\left(t_{n}\right)=y_{n}$. Then $t_{n} \leq t_{0}$ for large $n$. Now let $\tau$ be an accumulation point of $\left\{t_{n}\right\}$ in $\left[0, t_{0}\right]$. From $\gamma_{n}\left(t_{n}\right)=y_{n}$ and $\gamma_{n}(\tau) \rightarrow \gamma(\tau)$ it follows that $\gamma(\tau)=y$ as desired.

Before stating the main result of this section, we observe that one may assume WLOG that the geodesic segments $\gamma_{n}$ above contain $x_{n}$ and $y_{n}$ only as endpoints. Further, if each $\gamma_{n}$ contains a point $z_{n} \rightarrow z$ then $\gamma$ contains $z$. 
THEOREM 3.4. If $(X, \beta)$ is a principally causal and causally pseudocon-

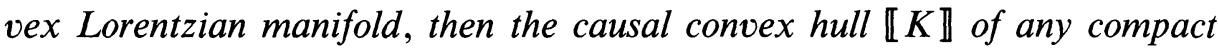
subset $K$ is compact.

Proof. By causal pseudoconvexity there is a compact set $K^{\prime}$ with $\llbracket K \rrbracket \subseteq K^{\prime}$. By Lemma 3.3, via the observation above, $\llbracket K \rrbracket$ is closed.

4. Lorentzian theory. In this section several known results for spacetimes are generalized to Lorentzian manifolds and new sufficient conditions are given for the existence of a causal geodesic segment joining two causally related points. According to the Hopf-Rinow Theorem [8, p. 163], in Riemannian manifolds completeness is a sufficient (but not a necessary) condition for the existence of a minimizing geodesic segment between any two points. A corresponding result for spacetimes is that global hyperbolicity is a sufficient (but not a necessary) condition for the existence of a maximizing geodesic segment between any two causally related points. (Meaning, of course, that each point is in the causal domain of the other). Since this existence is geometrically important, one is always interested in other sufficient conditions in the Lorentzian case. We shall obtain such conditions in terms of certain sectional curvatures in the presence of principal causality and causal pseudoconvexity.

We begin by considering distinguishing Lorentzian manifolds. Recalling the bicharacteristics of $\square-m^{2}$, small changes in the arguments on pp. 195-196 of [6] prove the following.

Proposition 4.1. If $(X, \beta)$ is a distinguishing Lorentzian manifold then it is principally causal.

We shall now define the (causal) distance function $d: X \times X \rightarrow \mathbf{R} \cup$ $\{\infty\}$. When necessary for clarity we denote it $d(\beta)$. Given $x \in X$, set $d(x, y)=0$ if $y \in X \backslash J(x)$, and for $y \in J(x)$ let $d(x, y)$ be the supremum of the lengths of all causal curves from $x$ to $y$. It is easy to see that $d$ is symmetric, non-negative, and satisfies a reverse triangle inequality (where appropriate). By adapting the proof on p. $215 \mathrm{f}$ of [6] it is easy to see that it is lower semicontinuous where it is finite.

Proposition 4.2. Let $d$ be the distance function of $(X, \beta)$.

(1) $X$ is chronological iff $d \mid \operatorname{diag}(X \times X) \equiv 0$.

(2) $X$ is distinguishing iff for all $x \neq y$ there exists $z$ such that exactly one of $d(x, z)$ or $d(y, z)$ is zero.

(3) $X$ is stably causal iff there exists a neighborhood $U$ of $\beta$ in the $C^{0}$-fine topology such that $d\left(\beta^{\prime}\right) \mid \operatorname{diag}(X \times X) \equiv 0$ for all $\beta^{\prime} \in U$. 
Proof. Ad 1: By definition, $X$ is chronological iff $x \notin I(x)$ for all $x \in X$, whence $d(x, x)=0$ for all $x$.

Ad 2: By definition, $X$ is distinguishing iff $x \neq y$ implies $I(x) \neq I(y)$. Thus we may assume that for all $x \neq y$ there exists $z \in I(x) \backslash I(y)$ such that $d(x, z)>0$ and $d(y, z)=0$. Conversely, the condition in 2 means we may assume that $I(x) \backslash I(y) \neq \varnothing$ whence $I(x) \neq I(y)$.

Ad 3: Combine 1 and the definition of stably causal.

In general, $d$ fails to be upper semicontinuous. Thus when $d$ is continuous it should imply restrictions on the causal structure of $X$. As an example, we have

TheOREM 4.3. Let $(X, \beta)$ be distinguishing. If $d$ is continuous then $X$ is causally continuous.

Proof. It suffices to show that $I$ is outer continuous. If not, then there exists a compact $K \subseteq X \backslash \overline{I(x)}$, for some $x \in X$, and a sequence $x_{n} \rightarrow x$ such that $K \cap I\left(x_{n}\right) \neq \varnothing$ for all $n$. Let $y_{n} \in K \cap I\left(x_{n}\right)$. By passing to a subsequence if necessary, we may assume that $y_{n} \rightarrow y \in K$. Since $X \backslash \overline{I(x)}$ is an open neighborhood of $y$ there exists $z \in X \backslash \overline{I(x)}$ with $z \in I(y)$. For sufficiently large $n, z \in I\left(y_{n}\right)$ and hence $z \in I\left(x_{n}\right)$. By changing the choice of $z$ if necessary, we may assume that $d\left(x_{n}, z\right) \geq d\left(x_{n}, y_{n}\right)+$ $d\left(y_{n}, z\right)$. Now $0<d(y, z) \leq \liminf d\left(y_{n}, z\right)$ so $d\left(x_{n}, z\right) \geq \frac{1}{2} d(y, z)>0$, eventually for all $n$. Since $d(x, z)=0, d$ is not continuous.

If a Lorentzian manifold is not time-orientable it has a double cover which is (cf. §2). The covering map is a local isometry, and two causally related points in the covering space can be joined by a causal geodesic iff their images below can. Using the covering map and simple lifting arguments it is not hard to show that one space is principally causal (causally pseudoconvex) iff the other space is principally causal (causally pseudoconvex). This covering space may be used to extend many results for spacetimes to Lorentzian manifolds which are not necessarily timeorientable.

We now consider the existence of causal geodesics joining given causally related points. If $(X, \beta)$ is a spacetime then each tangent space $T_{x} X$ has an induced Minkowski structure and time-orientation. A timelike plane in $T_{x} X$ is a two-dimensional linear subspace of $T_{x} X$ which has an induced Minkowski structure of signature zero $(+-)$. Each timelike plane is nondegenerate so there is a well-defined sectional curvature for timelike planes. Assume that $(X, \beta)$ has everywhere nonpositive timelike sectional 
curvature. Then there are no conjugate points along causal geodesics [4; 1]. Thus the tangent map $\exp _{x^{*}}$ of the exponential map at $x$ must be nonsingular at each causal $v \in T_{x} X$. Now $\exp _{x}$ maps a neighborhood of $v$ diffeomorphically onto a neighborhood of $\exp _{x}(v)$. Observe that there are well-defined future and past causal cones at each $v \in T_{x} X$ with respect to the Minkowski structure on $T_{x} X$. We shall show that for causal $v$, the image under $\exp _{x^{*}}$ of the future (past) causal cone at $v$ contains the future (past) causal cone at $\exp _{x}(v)$. Let $\tau_{v}: T_{x} X \rightarrow T_{v}\left(T_{x} X\right)$ be the canonical vector space parallel translation isomorphism [1, p. 260]. Via $\beta^{b}$ and $\tau_{v}$ we transport $\beta$ to $T_{v}\left(T_{x} X\right)$, obtaining $\bar{\beta}$; i.e., for $w \in T_{v}\left(T_{x} X\right)$,

$$
\bar{\beta}(w, w):=\beta\left(\beta^{b}\left[\tau_{v}^{-1}(w)\right], \beta^{b}\left[\tau_{v}^{-1}(w)\right]\right) .
$$

LEMMA 4.4. Let $(X, \beta)$ be a spacetime with everywhere nonpositive timelike sectional curvature. If $v$ is a future causal vector in $T_{x} X$, then under $\exp _{x^{*}}: T_{v}\left(T_{x} X\right) \rightarrow T_{\exp _{x}(v)} X$ the image of the future (past) causal cone at $v$ contains the future (past) causal cone at $\exp _{x}(v)$.

Proof. By continuity it suffices to consider timelike $v$. If $w \in T_{v}\left(T_{x} X\right)$ and $\tau_{v}^{-1}(w)$ is a future causal vector in $T_{x} X$, then it follows from Corollary 10.12 of $[1$, p. 338] that (even if $v$ is not a unit vector)

$$
\beta\left(\beta^{b}\left[\exp _{x^{*}} w\right], \beta^{b}\left[\exp _{x^{*}} w\right]\right) \leq \bar{\beta}(w, w) .
$$

When $w$ is an arbitrary future null vector at $v$ we obtain

$$
\beta\left(\beta^{b}\left[\exp _{x^{*}} w\right], \beta^{b}\left[\exp _{x^{*}} w\right]\right) \leq 0 .
$$

Thus the image under $\exp _{x^{*}}$ of the future causal cone at $v$ contains either the future or the past causal cone at $\exp _{x}(v)$. Since this must hold for all future timelike $v \in T_{x} X$, it follows from continuity that therefore the image under $\exp _{x^{*}}$ of the future causal cone at $v$ contains the future causal cone at $\exp _{x}(v)$.

We now establish the existence of timelike geodesic segments.

Proposition 4.5. Let $(X, \beta)$ be a principally causal and causally pseudoconvex Lorentzian manifold. If $X$ has everywhere nonpositive timelike sectional curvature then for each $z \in I(x)$ there exists a timelike geodesic segment from $x$ to $z$.

Proof. If suffices to consider spacetimes $X$. Then we may assume that $z \in I^{+}(x)$ and let $\gamma:[0, b] \rightarrow X$ be a future timelike curve from $x$ to $z$. For all small $t$ there is a future timelike geodesic from $x$ to $\gamma(t)$, and if 
there is a future timelike curve from $x$ to $\gamma\left(t_{0}\right)$ then the fact that $\exp _{x}$ is a local diffeomorphism on the future timelike cone implies the existence of future timelike geodesics from $x$ to $\gamma(t)$ for all $t$ near $t_{0}$.

By way of contradiction, suppose there exists a least value $\theta$ such that no future timelike geodesic from $x$ to $\gamma(\theta)$ exists. Let $t_{n} \rightarrow \theta^{-}$and as in the proof of Lemma 3.3 let $\gamma_{n}:\left[0, a_{n}\right] \rightarrow X$ be a sequence of timelike geodesic segments from $x$ to $y_{n}=\gamma\left(t_{n}\right)$. The sequence $\left\{\gamma_{n}\right\}$ has a limit curve which is a future causal geodesic from $x$ to $y=\gamma(\theta)$. It is in fact null by the definition of $\theta$. WLOG $\dot{\gamma}_{n}(0) \rightarrow v \in T_{x} X$ which is null and $\exp _{x}(a v)=y$ for some $a>0$, whence $a_{n} \rightarrow a$ and $a_{n} \dot{\gamma}_{n}(0) \rightarrow a v$. The relation $y \in$ $I^{+}\left(\gamma_{n}\left(a_{n}\right)\right)$ yields $\exp _{x}(a v) \in I^{+}\left(\exp _{x}\left[a_{n} \dot{\gamma}_{n}(0)\right]\right)$. Consequently, Lemma 4.4 implies that $a_{n} \dot{\gamma}_{n}(0)$ lies in the causal past of $a v$ in $T_{x} X$ for large $n$. But $a v$ is a future null vector and each $a_{n} \dot{\gamma}_{n}(0)$ is a future timelike vector.

We now state the main theorem of this section.

THEOREM 4.6. Let $(X, \beta)$ be a principally causal and causally pseudoconvex Lorentzian manifold. If $X$ has everywhere nonpositive timelike sectional curvature then for each $x \in X, J(x)$ is closed and for each $y \in J(x)$ there is a causal geodesic from $x$ to $y$ (degenerate if $x=y$ ).

Proof. Since $\overline{J(x)}=\overline{I(x)}$ it suffices to show that each point $y$ of $\overline{I(x)}$ can be joined to $x$ by a causal geodesic. There is a sequence in $I(x)$, $y_{n} \rightarrow y$. By Proposition 4.5 there is a causal geodesic $\gamma_{n}$ from $x$ to $y_{n}$ for each $n$. By Lemma 3.3 the limit curve of the sequence $\left\{\gamma_{n}\right\}$ exists and is a causal geodesic from $x$ to $y$.

Combining Proposition 4.1 and this theorem, we obtain

Proposition 4.7. Let $(X, \beta)$ be a distinguishing causally pseudoconvex Lorentzian manifold. If $X$ has everywhere nonpositive timelike sectional curvature then it is causally simple.

We conclude this section with a generalization to causally pseudoconvex spacetimes of a result for globally hyperbolic spacetimes. A spacetime is said to be future 1-connected iff any two future timelike curves from $x$ to $y$ are homotopic through future timelike curves with fixed endpoints $x$ and $y$. The proof of the following is similar to that of Corollary 10.16 of [1, p. 343] using Theorem 4.5 instead of global hyperbolicity for existence. 
Proposition 4.8. Let $(X, \beta)$ be a principally causal and causally pseudoconvex spacetime. If $X$ is future 1-connected with everywhere nonpositive timelike sectional curvature, then for all $x \in X$ and all $y \in I^{+}(x)$ there exists a unique (up to parametrization) future timelike geodesic from $x$ to $y$.

5. Warped products. In this section we establish sufficient conditions for a Lorentzian warped product (see \$2) to be principally causal and causally pseudoconvex. These yield a large class of causally pseudoconvex Lorentzian manifolds which are not globally hyperbolic.

If $\mathbf{R}^{n}$ is given the usual Euclidean metric $g_{0}$ and if $H$ is an open convex set in $\mathbf{R}^{n}$, then $\left(H, g_{0} \mid H\right)$ is a geodesically pseudoconvex Riemannian manifold. Clearly, geodesically pseudoconvex spaces need not be complete. The next example shows that, conversely, complete spaces need not be geodesically pseudoconvex.

EXAMPLE 5.1. If $\left(\mathbf{R}^{n}, g_{0}\right)$ is Euclidean, then there exists a Riemannian metric $g$ arbitrarily near $g_{0}$ in the $C^{0}$-fine topology such that $\left(\mathbf{R}^{n}, g\right)$ is not geodesically pseudoconvex. Let $K=\{x, y\}$ with $x=(1,0, \ldots, 0)$ and $y=$ $(-1,0, \ldots, 0)$. We make small changes in $g_{0}$ on each of the discs $D_{k}$ centered at $(0, k, 0, \ldots, 0)$ for $k=1,2,3, \ldots$ such that there is a geodesic from $x$ to $y$ which passes through $D_{k}$. Outside the $D_{k}$ the geodesics are the usual straight lines. In this way we can make $g, C^{0}$-fine close to $g_{0}$ but not $C^{1}$-fine close.

Let $X \times{ }_{\psi} H$ be a Lorentzian warped product of $(X, \beta)$ and $(H, h)$ with natural projections $\pi_{1}: X \times H \rightarrow X$ and $\pi_{2}: X \times H \rightarrow H$. A geodesic $\gamma ;(a, b) \rightarrow X \times_{\psi} H$ may be written as $\gamma=\left(\gamma_{1}, \gamma_{2}\right)$ where $\gamma_{l}=\pi_{i} \gamma$. From [1, p. 71] we obtain the equations which $\gamma_{1}$ and $\gamma_{2}$ satisfy:

$$
\begin{gathered}
{ }^{2} \nabla_{\dot{\gamma}_{2}} \dot{\gamma}_{2}=-\dot{\gamma}_{1}(\Psi) \dot{\gamma}_{2} ; \\
{ }^{1} \nabla_{\dot{\gamma}_{1}} \dot{\gamma}_{1}=\frac{1}{2} \frac{\operatorname{grad} \Psi}{\psi} \bar{h}\left(\dot{\gamma}_{2}, \dot{\gamma}_{2}\right) .
\end{gathered}
$$

Here ${ }^{1} \nabla$ and ${ }^{2} \nabla$ are the Levi-Civita connections on $(X, \beta)$ and $(H, h)$, respectively, and $\Psi=-\ln (-\psi)$. Equation (1) implies that $\gamma_{2}$ is a pregeodesic of $H$. Furthermore, if $\dot{\gamma}_{2}\left(t_{0}\right) \neq 0$ for some $t_{0}$ then $\dot{\gamma}_{2}$ is nonvanishing on $(a, b)$. In general $\gamma_{1}$ is not a pregeodesic of $X$. If the warping function $\psi$ is constant, however, both $\gamma_{1}$ and $\gamma_{2}$ are geodesics. Thus the geodesics of Lorentzian products $(\psi \equiv-1)$ are simply ordered pairs of geodesics. 
We now give some sufficient conditions for Lorentzian warped products to be principally causal and causally pseudoconvex which are independent of the warping function.

THEOREM 5.2. Let $(X, \beta)$ be a spacetime that is either globally hyperbolic or of the form $((a, b), \partial / \partial t \otimes \partial / \partial t)$ where $-\infty \leq a<b \leq \infty$. If $(H, h)$ is a geodesically pseudoconvex Riemannian manifold and $\psi$ is any warping function, then $X \times_{\psi} H$ is causally pseodoconvex and is stably causal, hence principally causal.

Proof. We shall assume that $(X, \beta)$ is globally hyperbolic. Then stable causality follows from the stable causality of $(X, \beta),[1$, pp. 32, 63].

Let $K \subseteq X \times H$ be compact. Then $\pi_{1}(K)$ and $\pi_{2}(K)$ are compact so there exist compact sets $K_{1}^{\prime} \subseteq X$ (by global hyperbolicity) and $K_{2}^{\prime} \subseteq H$ (by geodesic pseudoconvexity) such that any causal path in $X$ having both endpoints in $K_{1}$ lies in $K_{1}^{\prime}$ and any geodesic segment in $H$ having both endpoints in $K_{2}$ lies in $K_{2}^{\prime}$. Set $K^{\prime}=K_{1}^{\prime} \times K_{2}^{\prime}$. We claim that any causal geodesic segment of $X \times{ }_{\psi} H$ having both endpoints in $K$ lies in $K^{\prime}$.

Indeed, let $\gamma=\left(\gamma_{1}, \gamma_{2}\right):[a, b] \rightarrow X \times{ }_{\psi} H$ be a causal geodesic segment with $\gamma(a), \gamma(b) \in K$. Then $\gamma_{2}$ is a pregeodesic segment in $H$ with both endpoints in $K_{2}$, so it lies in $K_{2}^{\prime}$. Now $\gamma_{1}$ is a causal curve, not necessarily a pregeodesic, in $X$ with both endpoints in $K_{1}$, so it lies in $K_{1}^{\prime}$. Therefore $\gamma$ lies in $K^{\prime}$ and $X \times_{\psi} H$ is causally pseudoconvex.

Even is $H$ is not geodesically pseudoconvex it can happen that $X \times_{\psi} H$ is causally pseudoconvex, as will follow from Example 5.1 and this next result.

Proposition 5.3. Let $(X, \beta)$ be as in the preceding theorem. If $(H, h)$ is a complete Riemannian manifold and $\psi$ is any warping function, then $X \times_{\psi} H$ is globally hyperbolic, hence principally causal and causally pseudoconvex.

Proof. This follows from Theorems 2.53 and 2.54 of [1, p. 64-66].

In the special case of a Lorentzian product $(\psi \equiv-1)$ the globally hyperbolic condition on $X$ may be replaced with the weaker causal pseudoconvexity condition. 
THEOREM 5.4. Let $(X, \beta)$ be a causally pseudoconvex Lorentzian manifold. If $(H, h)$ is a geodesically convex Riemannian manifold then the Lorentzian product $(X \times H, \beta \oplus-h)$ is causally pseudoconvex. If $(X, \beta)$ is principally causal then the Lorentzian product is also.

Proof. The geodesics of the product are simply ordered pairs of geodesics of the factors. This, together with the techniques from the proof of Theorem 5.2, allow one to show that $(X \times H, \beta \oplus-h)$ is causally pseudoconvex. If $(X, \beta)$ is principally causal and $\gamma=\left(\gamma_{1}, \gamma_{2}\right)$ is a causal geodesic of the product, then $\gamma_{1}$ is a causal geodesic of $(X, \beta)$ which is not imprisoned there. It follows that $\gamma$ is not imprisoned in the product.

\section{REFERENCES}

1. J. Beem and P. Ehrlich, Global Lorentzian Geometry, New York: Marcel Dekker, 1981.

2. J. Beem, P. Ehrlich and T. Powell, Warped product manifolds in relativity, in Einstein volume, Athens: North Holland, 1982.

3. J. J. Duistermaat and L. Hörmander, Fourier integral operators II, Acta. Math., 128 (1972), 183-269.

4. F. Flaherty, Lorentzian manifolds of nonpositive curvature, in Proc. Symp. Pure Math. 27, part 2 (1975), 395-399.

5. F. G. Friedlander, The Wave Equation on a Curved Space-time, Cambridge, 1975.

6. S. Hawking and G. Ellis, The Large Scale Structure of Space-time, Cambridge, 1973.

7. S. Hawking and R. Sachs, Causally continuous spacetimes, Commun. Math. Phys., 35 (1975), 287-296.

8. N. Hicks, Notes on Differential Geometry, Princeton: Van Nostrand, 1965.

9. S. Lang, Differential Manifolds, Reading: Addison-Wesley, 1972.

10. B. O'Neill, Semi-riemannian Geometry, New York: Academic Press, 1983.

11. P. E. Parker, Distributional geometry, J. Math. Phys., 20 (1979), 1423-1426.

12. Mathematical Relativity, Syracuse University lecture notes, 1979.

13. M. Taylor, Pseudodifferential Operators, Princeton, 1981.

Received October 1, 1981.

UNIVERSITY OF MisSOURI-COLUMBia

COLUmbia, MO 65211

AND

UNIVERSITY OF IOWA

IOWA CITY, IA 52242 


\section{PACIFIC JOURNAL OF MATHEMATICS}

\section{EDITORS}

Donald BabbitT (Managing Editor)

University of California

Los Angeles, CA 90024

Hugo Rossi

University of Utah

Salt Lake City, UT 84112

C. C. Moore and Arthur Ogus

University of California

Berkeley, CA 94720
J. DugunduI

Department of Mathematics

University of Southern California

Los Angeles, CA 90089-1113

R. FinN and H. SAmelson

Stanford University

Stanford, CA 94305

\section{ASSOCIATE EDITORS}
R. ARENS
E. F. BECKENBACH
B. H. NEUMANN
F. WOLF
K. YosHIDA (1906-1982)

\section{SUPPORTING INSTITUTIONS}

UNIVERSITY OF ARIZONA

UNIVERSITY OF BRITISH COLUMBIA

CALIFORNIA INSTITUTE OF TECHNOLOGY

UNIVERSITY OF CALIFORNIA

MONTANA STATE UNIVERSITY

UNIVERSITY OF NEVADA, RENO

NEW MEXICO STATE UNIVERSITY

OREGON STATE UNIVERSITY
UNIVERSITY OF OREGON

UNIVERSITY OF SOUTHERN CALIFORNIA

STANFORD UNIVERSITY

UNIVERSITY OF HAWAII

UNIVERSITY OF TOKYO

UNIVERSITY OF UTAH

WASHINGTON STATE UNIVERSITY

UNIVERSITY OF WASHINGTON 


\section{Pacific Journal of Mathematics}

Vol. 107, No. $1 \quad$ January, 1983

John Kelly Beem and Phillip E. Parker, Klein-Gordon solvability and the

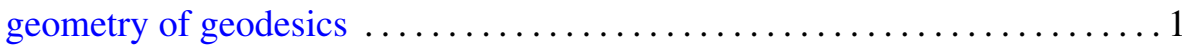

David Borwein and Amnon Jakimovski, Transformations of certain

sequences of random variables by generalized Hausdorff matrices ..... 15

Willy Brandal and Erol Barbut, Localizations of torsion theories . . . . . . . 227

John David Brillhart, Paul Erdős and Richard Patrick Morton, On sums

of Rudin-Shapiro coefficients. II ........................... 39

Martin Lloyd Brown, A note on tamely ramified extensions of rings $\ldots \ldots \ldots 71$

Chang P'ao Ch'ên, A generalization of the Gleason-Kahane-Żelazko

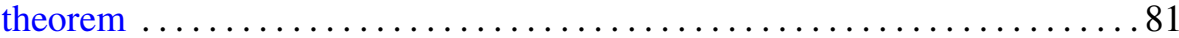

I. P. de Guzman, Annihilator alternative algebras $\ldots \ldots \ldots \ldots \ldots \ldots$. . . . 89

Ralph Jay De Laubenfels, Extensions of $d / d x$ that generate uniformly

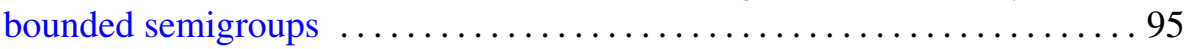

Patrick Ronald Halpin, Some Poincaré series related to identities of $2 \times 2$

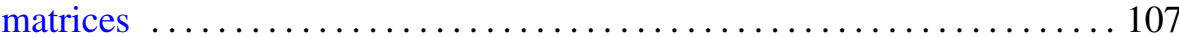

Fumio Hiai, Masanori Ohya and Makoto Tsukada, Sufficiency and

relative entropy in $*$-algebras with applications in quantum systems . . . 117

Dean Robert Hickerson, Splittings of finite groups $\ldots \ldots \ldots \ldots \ldots \ldots \ldots 14$

Jon Lee Johnson, Integral closure and generalized transforms in graded

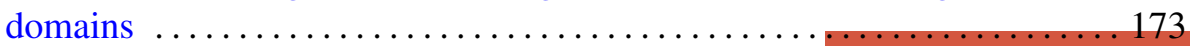

Maria Grazia Marinari, Francesco Odetti and Mario Raimondo, Affine

curves over an algebraically nonclosed field . ................. 179

Douglas Shelby Meadows, Explicit PL self-knottings and the structure of

PL homotopy complex projective spaces $\ldots \ldots \ldots \ldots \ldots \ldots \ldots \ldots \ldots$

Charles Kimbrough Megibben, III, Crawley's problem on the unique

$\omega$-elongation of $p$-groups is undecidable .................... 205

Mary Elizabeth Schaps, Versal determinantal deformations $\ldots \ldots \ldots \ldots 213$

Stephen Scheinberg, Gauthier's localization theorem on meromorphic

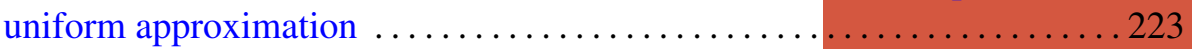

Peter Frederick Stiller, On the uniformization of certain curves . . . . . . . 229

Ernest Lester Stitzinger, Engel's theorem for a class of algebras . . . . . . . . 245

Emery Thomas, On the zeta function for function fields over $F_{p} \ldots \ldots \ldots 251$ 